BNL - 66450

\title{
FINAL REPORT \\ RELIABILITY TEST: \\ X-RAY RING EXIT CHAMBERS CROTCH RADIATION ABSORBERS
}

Donald R. Lynch and Jeffrey Morgan

National Synchrotron Light Source

April 9, 1999

\section{Brookhaven National Laboratory Operated by \\ Brookhaven Science Associates \\ Upton, NY 11973}

Under Contract with the United States Department of Energy

Contract Number DE-AC02-98CH10886 
DISCLAIMER

This report was prepared as an account of work sponsored by an agency of the United States Government. Neither the United States Government nor any agency thereof, nor any of their employees, nor any of their contractors, subcontractors or their employees, makes any warranty, express or implied, or assumes any legal liability or responsibility for the accuracy, completeness, or any third party's use or the results of such use of any information, apparatus, product, or process disclosed, or represents that its use would not infringe privately owned rights. Reference herein to any specific commercial product, process, or service by trade name, trademark, manufacturer, or otherwise, does not necessarily constitute or imply its endorsement, recommendation, or favoring by the United States Government or any agency thereof or its contractors or subcontractors. The views and opinions of authors expressed herein do not necessarily state or reflect those of the United States Government or any agency thereof. 


\begin{abstract}
This report details the efforts by engineers at the National Synchrotron Light Source (NSLS) of Brookhaven National Laboratory (BNL) to evaluate the reliability of water-cooled radiation absorbers used in the NSLS X-ray ring. These absorbers are part of the 16 dipole vacuum chambers which comprise the arc sections of the ring. They are located at the intersections (crotch) of the beamline exit ports with the electron beam chamber, and are commonly referred to as "crotches". The purpose of these efforts was to demonstrate the reliability of the crotches under operating conditions that the crotches will be subjected to over the entire expected life of the ring. The efforts described include engineering calculations, finite element analysis, conceptual design for a reliability test, test implementation and descriptions, results and conclusions related to these analyses and tests.
\end{abstract}

Keywords: crotch, thermal cycling, thermal stress, NSLS, X-ray ring 


\section{INTRODUCTION}

In March of 1993, in anticipation of RF cavity improvements which would allow the NSLS XRay Ring to be operated at its original design parameters of $500 \mathrm{mAmps}$ at $2.5 \mathrm{GeV}$ (or its thermal equivalent: $438 \mathrm{mAmps}$ at $2.584 \mathrm{GeV}$ ), then NSLS chairman Denis McWhan, created the NSLS X-Ray Ring Thermal Limits Task Force to investigate the ability of all ring components to operate reliably under these conditions. Prior to this time the ring had been unable to operate at greater than $250 \mathrm{mAmps}$ and $2.5 \mathrm{GeV}$.

Task Force chairman L. Berman and members N. Fewell, D. Lynch, P. Montanez, P. Stefan and J. Safranek identified 32 components (or, in some cases, classes of components) which are illuminated in some portion by either dipole magnet or insertion device radiation. The task force then determined whether passive protection through feedback interlocks was feasible, and, if not, thermal finite element analyses (FEA) were performed by task force members D. Lynch and P. Montanez. Based on these analyses the task force determined whether the component or class of component would operate reliably. Those cases where reliability could not be demonstrated in the initial analyses were then individually scrutinized and a suitable course of action was devised for each. The last of the components to be analyzed is the x-ray ring exit chamber crotch radiation absorber, more commonly referred to as simply the "crotch".

This report presents the significant results of this process as relates to the crotch. In this case, thermal and thermal stress analyses indicated potential problems which could not be dealt with analytically. While it was felt that the crotches would undergo minimal permanent deformation due to cyclic heat

loading, the Task Force believed that it was not great enough to cause any operational problems. In addition, the physical location of the crotch made it unacceptable to consider redesign and replacement (which would have resulted in the shutting down of the facility for an extended period of time). Consequently, it was decided to perform a simulation test of a representative part to demonstrate conclusively, that the crotch could perform its function reliably over the course of its expected lifetime. 


\section{SCOPE}

This report documents the tasks undertaken to demonstrate the reliability of the NSLS x-ray ring exit chamber crotch radiation absorbers (hereinafter referred to as "the crotch"), over the expected life of the x-ray ring. This work extended analyses performed and reported (see references in section 2.0 of this report) prior to these efforts. This report includes the following:

a. Mechanical analyses to characterize the cyclic thermal loading to which the subject crotch is expected to be exposed during the entire expected lifetime of the ring. These analyses build upon previous analytical efforts to devise a testing scheme which adequately and conservatively simulates the whole life of the crotch;

b. A description of the physical test devised in step a;

c. Results of the test described in step b;

d. Analyses and interpretation of the test results reported in step c;

e. Conclusions and recommendations based on the analyses of step d;

f. References, supporting data, and correlations as necessary to support the efforts described in steps a through e.

These analyses pertain only to the design of the subject crotches as they are installed in the NSLS X-ray ring. Generalization of the results and conclusions of the efforts described below to other applications and/or similar materials, components and installations are beyond the scope of this report. 


\section{MECHANICAL ANALYSES}

\section{Description of Crotch Absorber}

The crotch protects uncooled sections of the dipole vacuum chamber which would otherwise be illuminated by the $\mathrm{x}$-rays generated by the electron beam in the dipole magnetic field.

The electron beam follows a curved path in a magnetic field. When the electron beam curves it loses a small but finite quantity of energy which is manifested as photons. The specific quantity of energy thus

converted from electron momentum to photons determines the spectra in which the photons are manifested; i.e. x-rays for the NSLS dipole ring.

The photons thus generated are unaffected by the dipole magnet field and travel straight in the path that the electron beam was following when it emitted them, hence there are photons generated

continually in the arc the electron beam assumes due to the dipole magnetic field, tangentially from all points along that arc.

The chamber exit ports are two discrete openings in each dipole vacuum chamber, which allow photons to exit the ring and flow to the experimental beamlines. The areas immediately upstream of the first port, between the two ports and downstream of the second port are the uncooled sections of the dipole vacuum chamber and these are the areas that the crotches must protect.

The photons that the crotch shields from the vacuum chamber are absorbed in the crotch surface, and the energy of the photons is therein converted to heat. The heat is removed from the crotch surface by

conduction in the copper crotch to two integral cooling tubes.

The physical envelope of the crotch is designed to (a) fit within the confines of the dipole vacuum chamber, (b) allow the appropriate angular segment of the dipole radiation to pass through to the experimental beamlines, (c) maximize the surface area presented to the specific angular spread of photons that it absorbs and (d) optimize the conduction path from the absorbing surface to the cooling tubes.

\section{Analytical Rationale}

The actual electron beam parameters, in terms of beam current, are cyclical in nature, with the current maximized at each storage ring fill and decaying slowly for approximately 12 hours between fills. The consequential thermal effects that this has on storage ring components leads to a general thermal and stress analysis as follows: 
a. Identify all areas of the crotch which are irradiated by x-ray photons under any possible electron beam orbit.

b. Characterize the pattern of irradiation intensity and identify most severe exposures.

c. Perform steady state thermal analysis of most severe exposures to determine crotch temperature profile.

d. Perform transient thermal analysis to determine the time required for an irradiated crotch to return to ambient temperature when irradiation is discontinued.

e. Perform steady state thermal stress analysis to determine locations and magnitudes of highest stress concentration.

f. Perform thermal fatigue analyses to predict life expectancy of component under cyclical thermal loading.

The time determined in step $d$ represents $1 / 2$ of a thermal cycle (the other $1 / 2$ being the time required to reach steady state from ambient temperature when irradiation is reapplied).

Items a, b, c and d above have been completed and are reported separately in references [4], [6] and [8] of this report.

\section{Thermal Stress Analyses}

Thermal FEA was performed on each of the 3 crotch designs $\left(0^{\circ}, 2.5^{\circ}\right.$, and $\left.10^{\circ}\right)$ as reported in references [4] and [6] of this report. The FEA models constructed therein were then "cloned" to maintain geometric consistency, after which additional mechanical properties and mechanical boundary conditions were applied. Since the crotch is entirely constructed of OFHC copper, the pertinent properties are:

Modulus of Elasticity

Thermal Expansion Coefficient:

Poisson's Ratio:

Density:

Yield Strength (@705)

Yield Strength (@ 500%)
$1.7 \times 10^{7} \mathrm{psi}$

$9.6 \times 10^{-6} \mathrm{in} / \mathrm{in} /{ }^{\circ} \mathrm{F}$

.333

$.323 \mathrm{lbm} / \mathrm{in}^{3}$

$6,000-60,000$ psi

4,500 - 35,500 psi 
Boundary Conditions:

inclined
Fixed at all nodes on lowest plane of crotch block (on the flat face furthest of the model from the

(Note: Yield strength is greatly affected by the material hardness condition. Depending on the source for properties, there is a factor of as much as 10 (from fully hard to fully annealed) in yield strength. Since the crotches are machined from a hard material then brazed, the material properties are probably closer to the fully annealed condition. Most of the other properties listed above, however, do not vary greatly with the range of temperatures predicted by the thermal analyses nor with material hardness condition. The reported values provided for these properties are fairly consistent among a variety of sources.)

The "cloned" model then had the node temperatures set to those calculated for the respective thermal FEA models and 100 psi water pressure conditions applied to the cooling surfaces. The thermal stresses on the various crotches were then calculated using ALGOR ${ }^{\mathrm{TM}}$ FEA software. The resultant stress profiles for each of the three crotches are provided in Figures 1, 2 and 3.

(Note: The FEA models for the $2.5^{\circ}$ and $10^{\circ}$ crotches have had their cooling tube geometry modified and idealized as open rectangular channels with heat transfer properties and surface area equivalent to the actual round tubes. This was done for ease of extruding from $2 \mathrm{D}$ to $3 \mathrm{D}$ and provided for more accurate thermal and stress calculations of the stresses in the absorbing areas. Several iterations of more conservative partial models demonstrated the accuracy of these models in the areas of interest. The high stress on the cooling surfaces, however, can be ignored. These are artifacts of the model idealizations. The FEA meshing is highly refined in the areas of high thermal loads and much more coarse in other areas. A number of iterations were performed with variations in mesh density and geometry, and the models showed no significant difference when mapped using element averaged or nodal calculated values. The authors estimate that the accuracy of the FEA models are within $+/-20 \%$ in the relevant areas.)

The stresses over the bulk of the crotch models are well below the yield range, though the areas of highest stresses tend to coincide with areas of highest temperatures. Those areas undergo localized yielding. This would only be a problem if the yielding tended to cause a "runaway" effect (i.e. cause changes to the physical makeup and or properties of the crotch that tended to drive up temperatures and stresses which cause further physical and/or property changes, and so on). This does not seem likely, given the highly localized nature of the extreme temperatures and stresses, but it was not feasible to attempt to prove this analytically. 

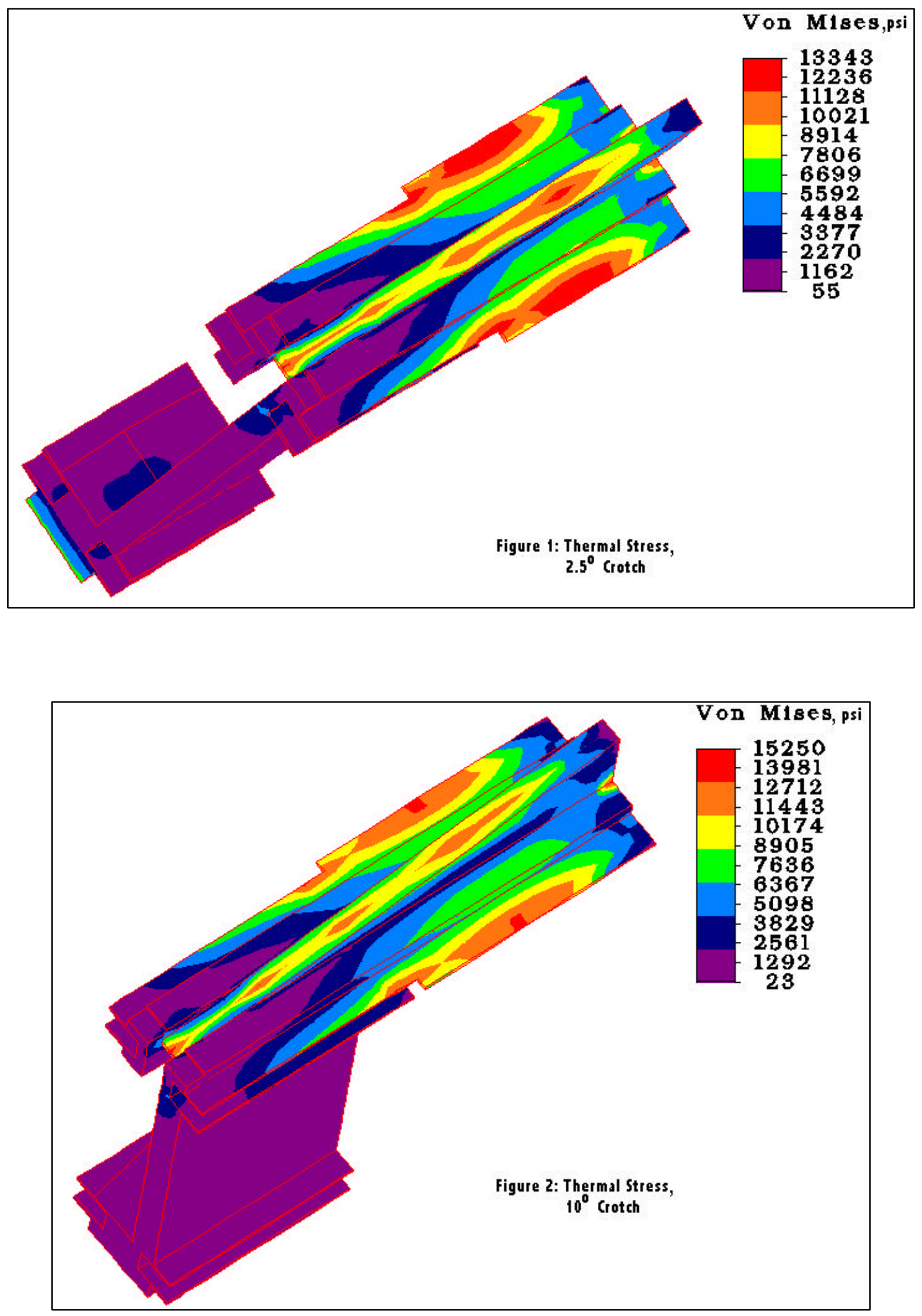


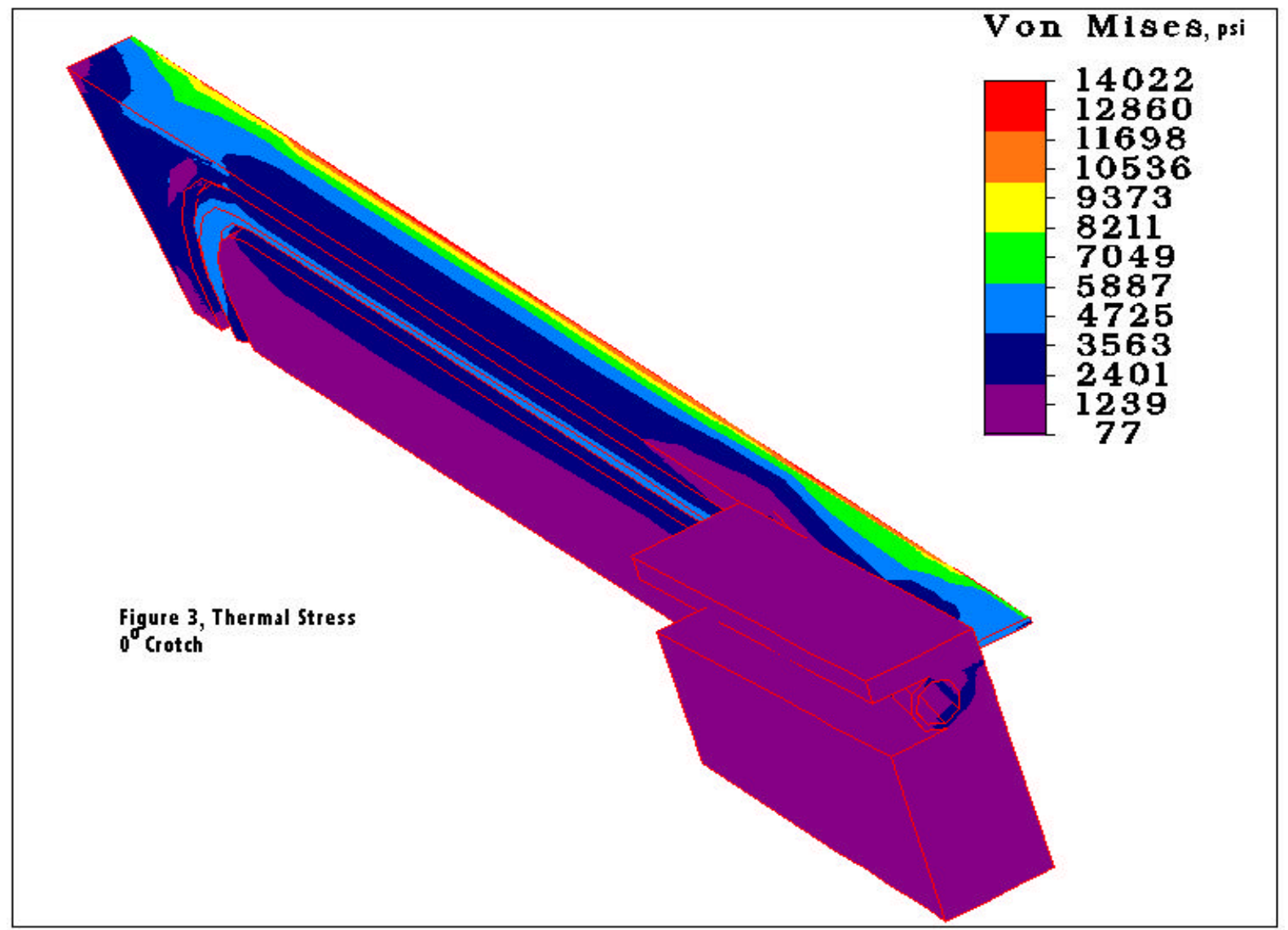

\section{Thermal Fatigue Analysis}

The results of the steady state stress analyses (maximum predicted stresses) were then used as the model stresses for a thermal cycling fatigue analysis. Data for OFHC fatigue stress, as was the case for yield strength data, varies quite a bit from source to source. Figure 4 provides a fatigue stress curve for two temperatures. This figure was constructed from a compilation of sources as a best engineering estimate for the values for the $70^{\circ} \mathrm{F}$ curve. The values for the $500^{\circ} \mathrm{F}$ curve were create by scaling the the $70^{\circ} \mathrm{F}$ values in accordance with the temperature dependence of yield strength. The $500^{\circ}$ curve was then used as the basis to calculate the number of cycles to failure.

It is obvious when comparing the maximum stresses calculated in the previous section of this report to the $500^{\circ} \mathrm{F}$ curve that none of the crotches can be expected to have an infinite life, where infinite life in this case, is defined as the absence of any localized yielding or permanent deformation for $1 \times 10^{7}$ thermal cycles. 


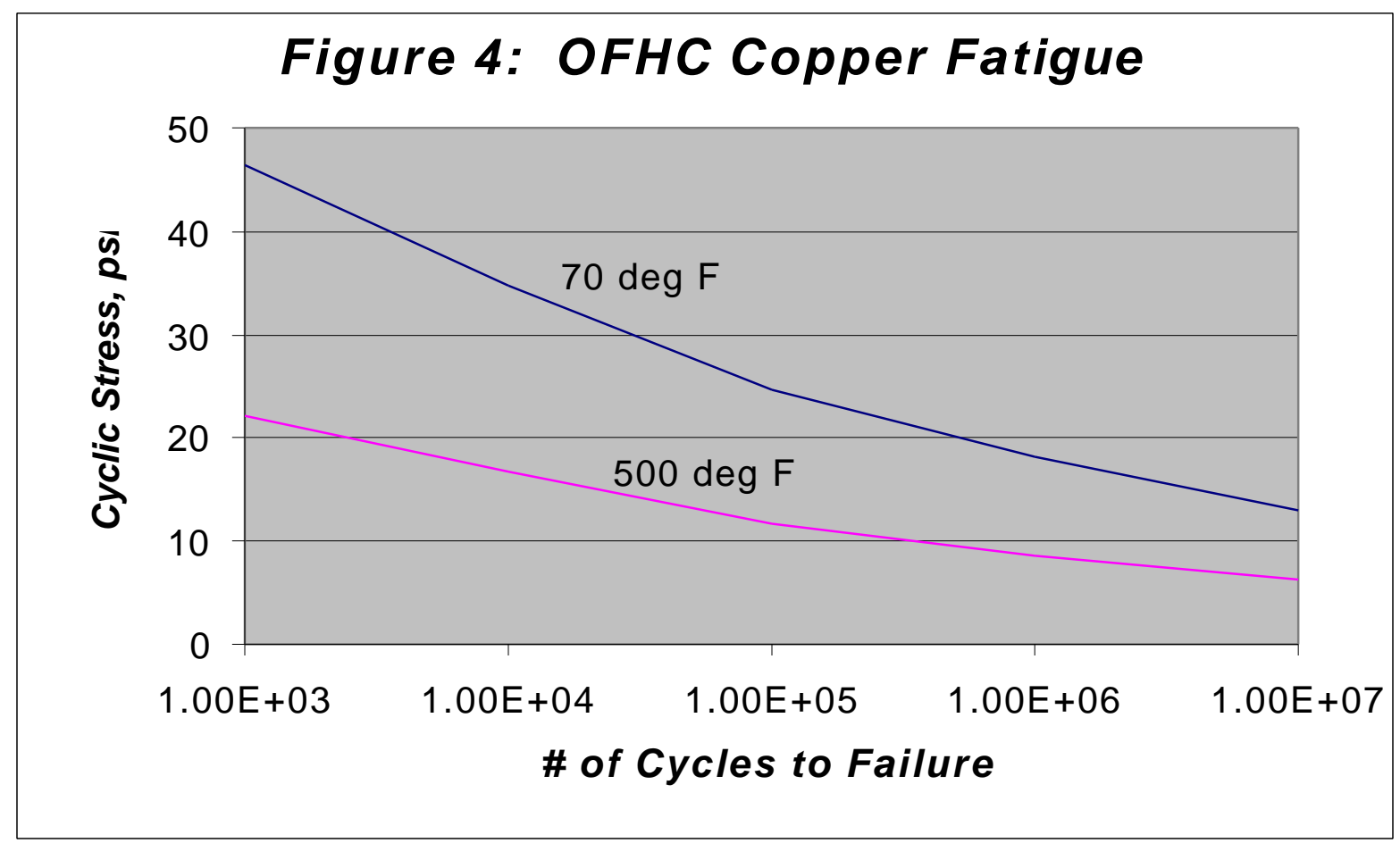

\section{Analyses Summary and Interpretation}

Table 1 provides a summary of the calculation results for the analyses described above.

The calculations performed tend to be conservative. The thermal loads used are based on worst case conditions. The thermal loads do not take into account mitigating effects such as radiation cooling and reflection, nor do they take credit for the fact that the stresses are not fully reversed. Conservative values for material and thermal transport properties are used in all calculations. The use of such conservative criteria allows for a greater degree of confidence that the calculation results don't over estimate the crotches useful lives.

The NSLS X-ray Ring has an expected lifespan of 20 years beyond the present. Given an average of 2 current fills per day and an average of 200 operating days per year, the crotches can be expected to experience a total of 8000 thermal cycles over the ring lifetime. Since the worst operating conditions experienced by the crotches from the time of original commissioning of the ring to the present are approximately $1 / 2$ the severity of the conditions used for the calculations in this report, the currently installed crotches are not suspected to have experienced any significant degradation of their life expectancies due to previously accumulated thermal cycles.

The fatigue analyses indicate a minimum expected life of 18,000 cycles $\left(10^{\circ}\right.$ crotch, others have longer expected lives). 


\section{Table 1: Calculation Summary}

$\begin{array}{llll}\text { Quantity } & \mathbf{0}^{\mathbf{0}} \text { Crotch } & \mathbf{2 . 5}^{\mathbf{0}} \mathbf{C r o t c h} & \mathbf{1 0}^{\mathbf{0}} \text { Crotch } \\ \text { beam angle intercepted } & 72.53 \mathrm{mrad} & 48.1 \mathrm{mrad} & 51.13 \mathrm{mrad} \\ \text { bend magnet flux } & 40 \mathrm{w} / \mathrm{mr} & 40 \mathrm{w} / \mathrm{mr} & 40 \mathrm{w} / \mathrm{mr} \\ \text { max. temperature } & 160.6^{\circ} \mathrm{C} & 246.9^{\circ} \mathrm{C} & 260.1^{\circ} \mathrm{C} \\ & \left(321^{\circ} \mathrm{F}\right) & \left(476.5^{\circ} \mathrm{F}\right) & \left(500.2^{\circ} \mathrm{F}\right) \\ \text { max. stress } & 96.6 \mathrm{MPa} & 91.8 \mathrm{MPa} & 105.6 \mathrm{MPa} \\ & 14.0 \mathrm{ksi} & (13.3 \mathrm{ksi}) & (15.3 \mathrm{ksi}) \\ \text { \# of max. stress cycles to failure } & 38,000 & 48,000 & 18,000 \\ \text { total absorbed heat } & 2901 \mathrm{watts} & 1924 \mathrm{watts} & 2045 \mathrm{watts}\end{array}$

There remain, however, some reservations concerning the accuracy of the stress and fatigue analyses.

There are some important observations that should be noted here:

a. Low cycle thermal fatigue is a poorly understood field. Thermal fatigue of materials is a combination of mechanical effects due to cyclic mechanical stress and material property changes due to temperature cycling. It is common engineering practice to assign a factor of safety of 20 to derate the calculated number of cycles to failure, in lieu of testing.

b. The above analysis attempts to address this problem by creating a more conservative curve for fatigue at a higher temperature. While this makes sense on a practical level, there is insufficient empirical data to support the accuracy of this assumption.

c. Fatigue curves represent empirical data for complete failure of a uniaxial loaded part subjected to uniform stress in the axis of interest. The nature of fatigue failure in a component such as the subject crotches, wherein there are large temperature and stress gradients, is far more complex. While a local area of the component might fail in some manner (e.g. develop a crack), it does not necessarily follow that such failure will propagate to the point that the component will fail to perform its intended function. 
d. It is the opinion of the authors of this report that, based on the above analyses and experience with similar materials under similar circumstances, there is a very low probability that the subject crotches will fail to function adequately for the expected life of the ring.

In light of these comments, it is reasonable to conclude that it would be prudent to demonstrate conclusively by a simulation test, that the crotches will be at least as reliable as the calculations in this report indicate. The next few sections of this report describe the formulation and implementation of such a test.

\section{TEST DESCRIPTION}

\section{General}

The temperature, stress and fatigue analyses above indicate that the crotch assemblies will function adequately for the expected lifetime of the ring. They do not, however, provide an adequate factor of safety to protect against the uncertainties in these analyses, particularly with respect to the thermal fatigue analysis. The analyses indicate that localized areas of the crotch might experience permanent deformation, but that these deformations would not be serious enough to affect reliable operation of the storage ring. These devices are installed in an extremely inaccessible location within the $\mathrm{x}$-ray ring. It is probable that an actual failure of one of these crotches would be no more costly in terms of machine downtime and replacement costs than any attempt to upgrade the existing crotches to a configuration which is unequivocally adequate for their intended service. Furthermore, there is no potential mode of failure of these components which would pose any safety risk, whatsoever, to personnel and/or the environment nor would cause any significant consequential damage to the facility. In order to eliminate any doubt about their reliability, however, NSLS engineering has decided that it would be most conclusive and cost effective to devise a simulated lifetime test of the crotch. The following sections of this report describe the concept and implementation of such a test.

\section{Test Concept}

As indicated above, the expected remaining life of the NSLS X-ray Ring encompasses a total of 8000 fill cycles. During each fill cycle, the power incident on the crotches is maximized after a fill is complete then decays over the next 12 hours until a subsequent fill. The effect of cyclic stress is largely unaffected by the time scale of the individual cycles, but rather the amplitude of stress variation and the total number of cycles are the important variables. Therefore, the basic concept for such a test requires only that the component experience the extremes of stress the full number of times it would experience those extremes in a real lifetime. The full number of cycles 
can then be run in as short a period as is required to fully heat and cool the test article to the desired extremes of temperature and stress. This allows a full lifetime of 20 years to be simulated in a much shorter period of time. The time needed to fully heat or cool the crotch was calculated in reference [8] of this report to be about 30 seconds. Since this time represents $1 / 2$ of a full cycle the simulation will be accurate with 1 minute cycles. The time to simulate 8000 cycles is therefore 8000 minutes or about 5.6 days (running continuously).

The next conceptual problem for this test is how to apply an equivalent heat load over the appropriate area of the crotch absorbing surface. The x-rays absorbed by the crotch are absorbed and converted to heat very near the crotch absorbing surface. An electron beam of equivalent power directed and distributed across the same surface area would be absorbed even closer to the surface, allowing for a slightly conservative simulation. Peter Stefan describes how this could be accomplished in his NSLS Tech Note [7]. As indicated therein, either a custom designed electron gun or an electron beam welding machine (EBW) with appropriate controls could perform the simulation.

The final conceptual hurdles for the simulation test are the specifications of the test article and the power loading. In examining the temperature and stress analyses for the 3 crotch designs, it is clear that in terms of temperature and stress the $10^{\circ}$ crotch experiences the most severe loading. It would make sense then to use a $10^{\circ}$ crotch and its worst power loading as the test specifications. No $10^{\circ}$ crotch was available, however, while a spare $2.5^{\circ}$ crotch was available. This crotch had been manufactured at the same time as those currently installed, which gives the added benefits of randomness in its selection and assurance that its material properties are identical to those in service. The geometry of the $2.5^{\circ}$ crotch is very similar to the $10^{\circ}$ version, and the differences in the thermal and stress calculations is mostly due to the differences in the geometry of the thermal loading. In order to simulate the stresses on the $10^{\circ} \mathrm{crotch}$, it is then necessary to scale the power applied by some conservative relationship. Since the variation in maximum stress represented the greatest scaling ratio ,this was used and the power applied to the test crotch was specified as 2500 watts. [9]

\section{Preliminary Tests}

In order to verify the ability of an EBW machine to simulate the power density loading of the Xray ring photon beam, preliminary tests were run at the BNL EBW center. These tests were performed on a separate test article, which had geometric features which closely simulated those of the actual crotch, but was not water cooled. During these tests the beam angle of incidence, beam current, beam

potential and focussing potential were varied to find a suitable combination which would, as closely as possible, evenly distribute the beam on the absorbing surface and maintain a maximum beam width of .008 inches (which is the nominal width of the x-ray photon beam when it reaches the crotch surface), with as small a deviation as possible from this width across the entire length of the absorbing surface.

The results indicated optimal simulation with beam current $=16.7 \mathrm{mAmps}$, beam potential $=150$ $\mathrm{kVolts}$, and angle of inclination (of the absorbing surface to the centerline of the electron beam) 
$=45$ degrees. This provided the 2500 watts with maximum beam width of .008 in at either end of the absorbing surface and a minimum width of .006 in at the center of this surface. (Note: the focussing potential is a small variable electric potential which is applied to the beam but does not effect its power. The EBW has a variable control with a scalar readout which is dimensionless.

As indicated above, the power simulation required is the power generated by the ring when operating at $438 \mathrm{mAmps}$ and $2.584 \mathrm{GeV}$ (or its thermal equivalent). In this operating condition it has been determined [9] that, under worst case loading, the simulated crotch would absorb 2500 watts along its inclined absorbing surface. The EBW equipment will generate an equivalent amount of power when the product of its beam potential and current is equal to 2500 watts.

\section{Thermal Cycling Fatigue (Reliability) Test}

Due to scheduling conflicts, the EBW at BNL was unavailable for the full reliability test. The test was, instead, performed under contract by PTR-Precision Technologies, Inc. of Enfield, CT. PTR is a leader in the field of EBW technologies. They manufacture, repair, upgrade and service EBW machines, and perform manufacturing and testing contract work. They were well equipped for the task and performed all of the test requirements per the BNL test specification [9].

In order to simulate the distribution of x-ray photons on the absorbing surface, the EBW has a pattern generator which can rapidly scan the electron beam over a surface in a variety of patterns. For the subject test the, beam was swept back and forth in a straight line along the absorbing surface of the crotch at a cyclical rate of $100 \mathrm{hz}$ for a half cycle time of 30 seconds, then the beam was turned off for the other half cycle. This was repeated 8000 times to simulate a complete thermal lifetime of the crotch. Additional margin of safety was incorporated into the test procedure by requiring that an additional 1000 cycles at 3000 watts be performed on the test article.

Successful completion of the test was defined as completing all of the prescribed cycles without becoming deformed or damaged to the extent that water leaks of any magnitude are detected or the ability of the crotch to perform its heat absorbing function is in any way compromised.

\section{TEST RESULTS}

The reliability test described above was performed at PTR facilities in Enfield, CT, between $12 / 15 / 98$ and 1/25/99. Prior to commencement of the test the test article was examined using dye penetrant inspection techniques at BNL to establish a baseline condition of the item and to assure that the item was free from any pre-existing defects including cracks, surface imperfections and manufacturing deficiencies. Dye penetrant tests were again performed, at PTR, near the midpoint of the 8000 cycle full power test and between the 8000 cycle full power test and the 1000 cycle test at $120 \%$ full power. After completion of all cycling tests the test article was returned to BNL where a final dye penetrant test was performed. All of these tests showed no indication of significant degradation of the test article, in terms of cracks, deformation or erosion. On the 
surface there was evidence of grain growth and recrystallization, especially at the nose, but highly localized and nothing which might indicate an impending failure.

In addition to the dye penetrant tests, 4 thermocouples were mounted on the test article. These thermocouples were monitored continuously over the entire test. Two of the thermocouples were used for monitoring the water in and out temperatures, while the other 2 were mounted on surfaces of the crotch near expected hot zones. Since it was not possible to measure temperature at the zones where maximum temperatures were expected (from the above analyses) without affecting the test, the absolute magnitude of these temperatures was not meaningful, but rather they were made available to monitor relative changes. A relative change in the cyclic pattern of these temperatures from cycle to cycle would indicate the test article undergoing permanent changes induced by thermal fatigue. No significant changes were noted over the entire test.

Figure 5 shows the profile of these temperatures for a typical cycle, relative to the EBW current. In the figure, "nose" refers to the leading edge of the absorbing surface while "tail" refers to the trailing edge.

Figure 5: NSLS Exit Chamber Crotch Radiation Absorber Reliability Test, Typical Cycle

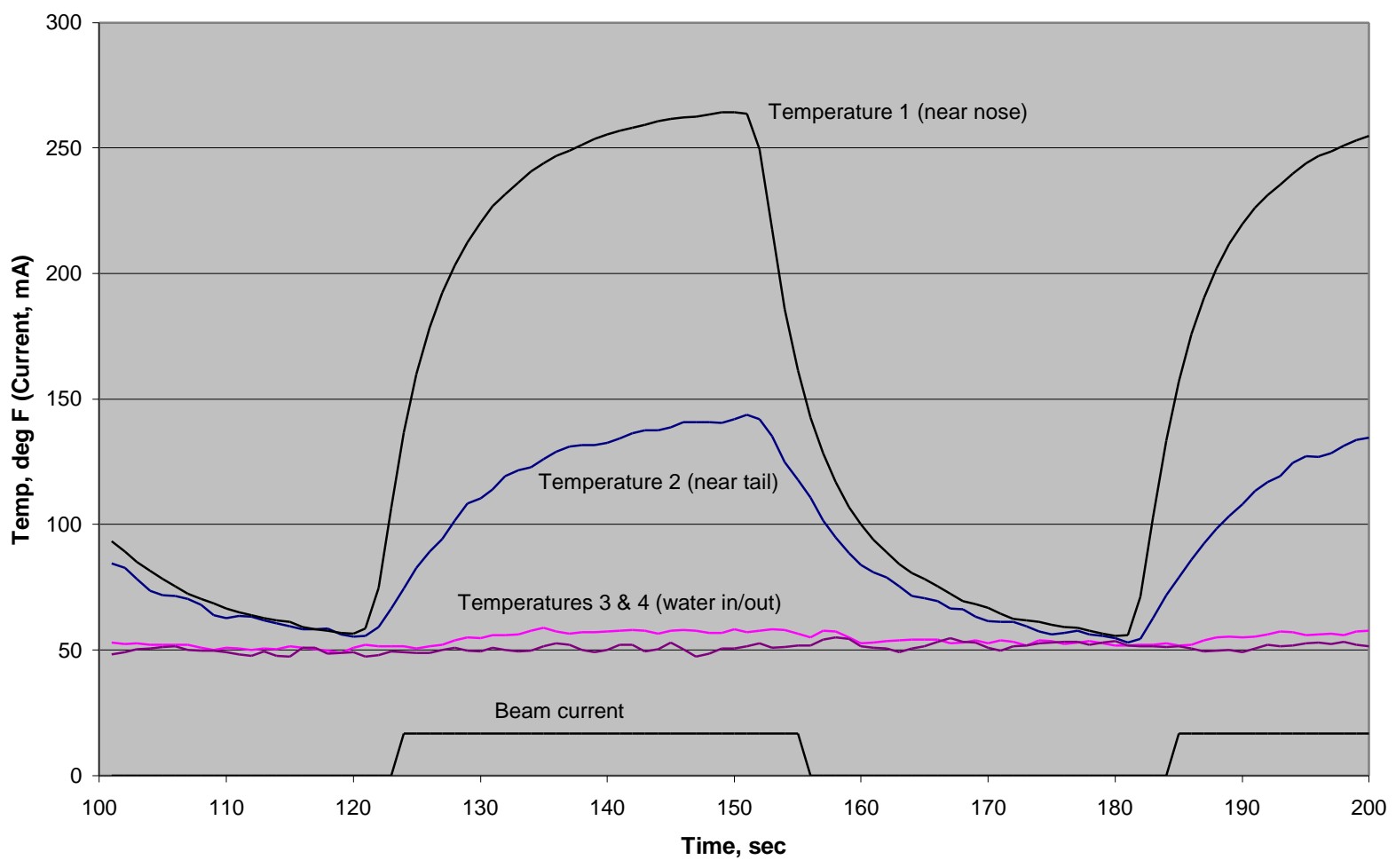




\section{INTERPRETATION OF TEST RESULTS}

The results of the dye penetrant tests showed no significant change to the test article and no evidence of impending failure. The only effects noted were surface effects highly localized on the surface area directly in the path of the electron beam. This suggests that the test article survived the full life cycle test without degradation in its ability to perform its function and that significant additional life beyond its required life could be expected.

Temperatures monitored during the test did not vary greatly from any one cycle to any others . The small variations which were detected were random and tracked with minor variations in water temperature and water flow. The absolute temperatures measured on thermocouples mounted to the crotch body were consistent with temperatures expected at those locations from analytical calculations. This suggests that the test was an adequate simulation of the life that these crotches are expected to endure over the expected life of the x-ray ring, if operated consistently at its design conditions.

Temperatures monitored for the water in and out indicated that the heat load being absorbed by the cooling water was consistent with the applied heat load within the expected margin of error (due to radiation losses, instrumentation tolerances and minor variations in water flow). This also indicates an adequate simulation of the actual service conditions.

\section{CONCLUSIONS}

Based on the analyses and tests reported herein, the authors have reached the following conclusions:

a. The test run was an adequate, accurate and conservative simulation of the thermal environment that the crotch is expected to experience over its projected lifetime. (Adequate because it provides the thermal load to the crotch in good approximation of its actual exposure in the storage ring, accurate because the size, shape and intensity of the e-beam closely simulates the xray beam parameters with respect to the crotch absorbing surface under the storage ring design conditions, and conservative because the worst case assumptions were used throughout, and an additional 1000 cycles at $120 \%$ of the maximum expected power were performed.)

b. The crotch survived the test with no significant degradation in its capability to absorb x-ray radiation. This is clearly demonstrated by physical examination, dye penetrant inspection, and examination of the data collected which indicates no measurable changes in the thermal profile of the crotch from the first cycle to the last.

c. The close correspondence between calculated values of temperature and cycle times and those measured by test validates the FEA models and methods used. 
d. The results validate the fatigue analyses described in this report.

e. It is recommend that any restriction or limits on x-ray storage ring operating conditions based on the questionable reliability of the crotch be removed. There is now no reason to suspect that the crotch will not perform its required function under maximum operating conditions for and beyond the design lifetime of the storage ring. 


\section{Acknowledgements}

The authors would like to thank Lonny Berman, Paul Montanez, Peter Stefan and Marty Woodle for their support and contributions to this report. This work was supported by the U.S. Department of Energy under contracts DE-AC0276CH00016 and DE-AC02-98CH10886.

\section{REFERENCES}

1. "Crotch Assembly (2.5 Beam Port)", ", Brookhaven National Laboratory Dwg. \#SLS-35.379-3A, Aug. 1980

2. "Crotch Assembly (10 $10^{\circ}$ Beam Port)", Brookhaven National Laboratory Dwg. \#SLS-35.3-78-4A , Oct. 1980

3. "Crotch Assembly ( $0^{\circ}$ Beam Port)", Brookhaven National Laboratory Dwg. \#SLS-35.3-3553A, Sep. 1987

4. D.R. Lynch, "Thermal Finite Element Analysis of X-ray Ring Bend Magnet Crotches", Brookhaven National Laboratory, internal report, Dec. 1994

5. D.R. Lynch, P.A. Montanez, "500 mAmp Task Force Analysis Summary", Brookhaven National Laboratory, <unpublished internal report>, Dec. 1994

6. D.R. Lynch, "Thermal Finite Element Analysis of X-ray Ring Phase II Bend Magnet Crotch Absorbers", Brookhaven National Laboratory, internal report, Jun., 1995

7. P.M. Stefan, "X-ray Ring Crotch Thermal Fatigue Test: First Cut Electron Gun Design" Brookhaven National Laboratory/National Synchrotron Light Source Technical Note \#514, May 1998

8. J. Morgan, D. Lynch, M. Woodle, "Thermal Cycle Time Simulation Analysis of a Crotch Photon Absorber in an X-Ray Storage Ring", Brookhaven National Laboratory \#65831, Aug. 1998

9. D.R.Lynch, "Specification For X-ray Ring Exit Chambers Crotch Reliability Test", Brookhaven NationalLaboratory Spec. \# SLS-07.144-001A, Oct. 1998

10. Holman, J.P. Heat Transfer, 3rd Ed., McGraw-Hill, New York, 1972

11. Baumeister (Ed.), Mark's Standard Handbook for Mechanical Engineers, 8th Ed., McGrawHill, New York, 1978

12. Technical Survey, OFHC Brand Copper, High Conductivity, Oxygen Free, The American Metal Company, New York, 1957

13. Metals Handbook, 9th Ed., American Society for Metals (ASM), Metals Park, OH, 1979 